\title{
DETERMINAÇÃO RÁPIDA DE HIDROQUINONA USANDO ANÁLISE POR INJEÇÃO EM BATELADA (BIA) COM DETECÇÃO AMPEROMÉTRICA
}

Rafael Rodrigues Cunha, Thiago Faria Tormin, Eduardo Mathias Richter e Rodrigo Alejandro Abarza Munoz*

Instituto de Química, Universidade Federal de Uberlândia, Av. João Naves de Ávila, 2121, 38400-902 Uberlândia - MG, Brasil

Recebido em 13/8/12; aceito em 19/11/12; publicado na web em 28/2/13

FAST DETERMINATION OF HYDROQUINONE BY BATCH INJECTION ANALYSIS (BIA) WITH AMPEROMETRIC DETECTION. A fast analytical method for determination of hydroquinone in pharmaceutical formulations employing batch injection analysis (BIA) with amperometric detection using a boron-doped diamond electrode is described. The supporting electrolyte was a $0.1 \mathrm{~mol} \mathrm{~L}^{-1} \mathrm{H}_{2} \mathrm{SO}_{4}$ solution (the single reagent used for analysis). The method showed good repeatability (RSD of $0.45 \%$, $\mathrm{n}=20$ ), wide linear range (from 10 to $\left.2000 \mu \mathrm{mol} \mathrm{L}^{-1}, \mathrm{R}=0.9999\right)$, low detection limit $(0.016 \mu \mathrm{mol} \mathrm{L}-1)$ and satisfactory recovery values $(91-96 \%)$. Accuracy of the method was evaluated by comparative analyses using high-performance liquid-chromatography. The ability to replace the electronic pipette by disposable syringes (injection procedure) in BIA systems was also shown.

Keywords: amperometry; BIA; boron-doped diamond (BDD).

\section{INTRODUÇÃO}

O composto 1,4-benzenodiol, comumente conhecido como hidroquinona (HQ), é o agente despigmentante de pele mais usado topicamente no tratamento de hipermelanoses como o cloasma, dermatite de berloque, hiperpigmentação pós-inflamatória, entre outras manchas dermatológicas. Tem ação de inibir a produção de melanina, composto que dá a coloração cutânea. Geralmente é encontrado associado a outros princípios ativos como, por exemplo, ácido retinoico, ácido glicólico, bissulfito de sódio (BS) e ácido ascórbico (AA), na forma de géis, loções ou pomadas cremosas, tanto em cosméticos industriais quanto em manipulados. ${ }^{1}$

A concentração usual de hidroquinona varia de 2 a $10 \%(\mathrm{~m} / \mathrm{m})$ e pode ocasionar irritação na pele, como eritema ou até mesmo erupções. Por ser um composto fenólico de fácil oxidação, geralmente são usados antioxidantes para evitar o escurecimento da formulação. Em meio básico, sua oxidação é instantânea e resulta em um composto amarelo escuro (respectiva quinona). Dentre os antioxidantes mais utilizados em meio aquoso, destacam-se o BS, ditionito de sódio ou combinações destes com butil-hidroxi-tolueno (BHT) e AA em formulações oleosas.

Diferentes métodos analíticos visando a determinação de hidroquinona em formulações farmacêuticas foram reportados usando espectrofotometria, ${ }^{2}$ espectrofluorimetria, ${ }^{3}$ cromatografia líquida de alta eficiência (HPLC), ${ }^{4}$ cromatografia em camada delgada ${ }^{5}$ e eletroforese capilar. ${ }^{6}$ Métodos eletroanalíticos também foram descritos para a determinação de hidroquinona empregando principalmente eletrodos modificados. ${ }^{7-14}$ Sob nosso conhecimento, há poucos estudos sobre a eletro-oxidação de HQ em eletrodo de diamante dopado com boro, ${ }^{15-17}$ sendo que nenhum deles teve como objetivo a aplicação analítica do eletrodo.

Nos últimos anos, o eletrodo de diamante dopado com boro (BDD, Boron Doped Diamond) tem sido usado em diferentes aplicações, como na desinfecção eletroquímica de águas (oxidação de micro-organismos ou matéria orgânica) e no desenvolvimento de novos métodos eletroanalíticos. O sucesso deste material se deve a diversas características, como superfície inerte, corrente de fundo baixa e estável, e ampla faixa de trabalho com estabilidade por

*e-mail: raamunoz@iqufu.ufu.br longos períodos de análise (menor susceptibilidade a fenômenos de adsorção). ${ }^{18-20}$

Como a hidroquinona é utilizada largamente em produtos cosméticos, o desenvolvimento de métodos de análise que apresentem características como simplicidade, rapidez, eficiência, seletividade, baixo custo e baixo consumo de reagentes é de extremo interesse em laboratórios de controle de qualidade. Métodos analíticos empregando detectores amperométricos acoplados a sistemas de análise por injeção em batelada (BIA, Batch Injection Analysis) apresentam tais características. ${ }^{21}$ Em comparação aos métodos analíticos que empregam o sistema de análise por injeção em fluxo (FIA), os métodos que empregam o sistema BIA têm como vantagens adicionais a eliminação de problemas típicos relacionados às válvulas e bombas do sistema FIA, a eliminação da solução transportadora de reagente e a possibilidade do desenvolvimento de métodos analíticos com

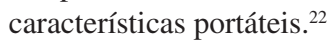

Neste trabalho apresenta-se o desenvolvimento de um método simples, rápido e de baixo custo empregando eletrodo de BDD acoplado a um sistema BIA para determinação amperométrica de hidroquinona em formulações cosméticas.

\section{PARTE EXPERIMENTAL}

\section{Reagentes e amostras de hidroquinona}

Água deionizada de alta pureza $(\mathrm{R} \geq 18,2 \mathrm{M} \Omega$-cm) obtida de um sistema de purificação de água Milli Q (Millipore, Bedford, MA, USA) foi usada na preparação de todas as soluções aquosas. O hidróxido de sódio $(98 \%, \mathrm{~m} / \mathrm{m})$ utilizado foi obtido da Dinâmica (São Paulo, Brasil). Ácido sulfúrico (95\%, m/v), ácido acético (65\%, $\mathrm{m} / \mathrm{v}$ ), ácido fosfórico $(85 \%, \mathrm{~m} / \mathrm{v})$ e acetato de sódio foram obtidos da Vetec (Rio de Janeiro, Brasil) e foram usados sem prévia purificação. Os reagentes acima listados foram usados como eletrólito suporte (0,1 mol L-1) para os estudos empregando voltametria hidrodinâmica. Hidroquinona ( $99 \%$ m/m, HQ), bissulfito de sódio (BS) e ácido ascórbico (AA) foram obtidos da Vetec (Rio de Janeiro, Brasil). O padrão de HQ ficou armazenado em refrigerador a cerca de $5{ }^{\circ} \mathrm{C}$. Soluções padrão de trabalho foram preparadas pouco antes das análises, usando diluição apropriada da solução estoque no eletrólito suporte. A solução padrão estoque de $\mathrm{HQ}\left(10 \mathrm{mmol} \mathrm{L}^{-1}\right)$ foi preparada 
em água deionizada. As amostras do creme cutâneo foram adquiridas numa drogaria local e fortificadas com os antioxidantes BS e AA, simulando amostras manipuladas. A amostra foi preparada mediante dissolução de uma massa adequada do creme em água e posterior manutenção em banho ultrassônico por $15 \mathrm{~min}$.

\section{Medidas eletroquímicas}

As medidas eletroquímicas foram conduzidas usando um potenciostato $\mu$-Autolab Type III (EcoChemie, Utrecht, Holanda). Injeções de solução padrão ou amostra foram conduzidas usando uma micropipeta eletrônica Eppendorf (Multipette ${ }^{\circledR}$ stream), que permite injeções com volumes $(10$ a $1000 \mu \mathrm{L})$ e velocidades (de 28 a $250 \mu \mathrm{L} \mathrm{s}^{-1}$ ) variáveis usando uma ponteira Combitip ${ }^{\circledR}$ de $1 \mathrm{~mL}$. Alternativamente, uma seringa descartável (BD Ultra-Fine II; 0,3 $\mathrm{mL}$ ) normalmente usada na aplicação de insulina foi usada no procedimento de injeção do sistema BIA.

A célula BIA construída em laboratório foi apresentada em trabalhos anteriores. ${ }^{23-25}$ Essa célula possui um volume interno de $180 \mathrm{~mL}$ e foi construída a partir de um cilindro de vidro (diâmetro interno de $7 \mathrm{~cm}$ ) e duas tampas de polietileno, que foram firmemente fixadas no topo e na base do cilindro. A tampa superior contém três orifícios para os eletrodos auxiliar, de referência e para a ponteira da micropipeta (Combitip ${ }^{\circledR} \mathrm{em}$ forma de seringa). A ponteira da micropipeta (com diâmetro externo de $6 \mathrm{~mm}$ ) foi firmemente introduzida no orifício central da tampa (diâmetro de $6,1 \mathrm{~mm}$ ), tornando o procedimento de injeção altamente reprodutível. Quando a seringa descartável foi empregada no procedimento de injeção, a tampa superior foi substituída por outra onde o orifício central era compatível com o diâmetro externo da seringa $(5,9 \mathrm{~mm})$. A tampa inferior da célula contém um único orifício (que também foi posicionado no centro da tampa) onde o eletrodo de trabalho de BDD foi posicionado (placa de $0,7 \times 0,7 \mathrm{~cm}$ ). A área do eletrodo foi delimitada por um $O$-ring de borracha $\left(0,500 \mathrm{~cm}^{2}\right)$. O contato elétrico foi feito com uma placa de cobre posicionada abaixo do eletrodo de BDD. Nesta disposição, durante a injeção, a ponteira da micropipeta fica posicionada a aproximadamente $2 \mathrm{~mm}$ da superfície do eletrodo de trabalho em uma configuração do tipo wall-jet. O sistema de agitação da solução na célula BIA foi implementado empregando-se um micromotor DC de $12 \mathrm{~V}$ retirado de uma máquina de xerox. A velocidade de agitação, neste caso, pode ser controlada mediante o uso de pilhas comuns, o que permite o uso da célula com agitação de forma portátil. Maiores detalhes sobre o sistema de agitação podem ser encontrados em trabalho publicado anteriormente. ${ }^{22}$ No presente trabalho, a velocidade de agitação utilizada foi de $2600 \mathrm{rpm}$.

Os eletrodos de referência e auxiliar foram um eletrodo miniaturizado $\mathrm{Ag} / \mathrm{AgCl}(\mathrm{KCl} \text { saturado })^{26} \mathrm{e}$ um fio de platina, respectivamente. Um filme fino (de aproximadamente 1,2 $\mu \mathrm{m}$ ) de diamante dopado com boro (ao redor de $8000 \mathrm{ppm}$ ) depositado sob um subtrato de silício policristalino de $1 \mathrm{~mm}$ de espessura $(0,7 \times 0,7 \mathrm{~cm})$ foi usado como eletrodo de trabalho (Adamant Technologies SA, La Chauxde-Fonds, Suíça). O custo de uma placa de silício de 0,7 x $0,7 \mathrm{~cm}$ é de aproximadamente R \$30,00 ( US\$15.00), sendo que a mesma placa foi usada em todos os estudos. Antes do eletrodo de BDD ter sido usado pela primeira vez, dois tipos de limpezas eletroquímicas (ativações) foram realizados, a anódica $(+2,6 \mathrm{~V}$, durante $900 \mathrm{~s}$ em meio de tampão Britton-Robinson $)^{27}$ e posteriormente a catódica (-3, 0 V durante $900 \mathrm{~s}$ em meio de $\left.\mathrm{H}_{2} \mathrm{SO}_{4} 0,2 \mathrm{~mol} \mathrm{~L}^{-1}\right) .{ }^{18}$ Diariamente, antes do início das análises, apenas a limpeza catódica era efetivamente realizada. ${ }^{18,28-30} \mathrm{~A}$ limpeza anódica somente é necessária quando ocorre uma contaminação mais drástica do eletrodo de BDD, o que não ocorre no procedimento proposto de determinação de HQ. Eletrodos comerciais de ouro $\left(0,283 \mathrm{~cm}^{2}\right.$; Metrohm) e carbono vítreo $(0,283$ $\mathrm{cm}^{2}$; Metrohm) foram usados em estudos preliminares. Antes do uso, um polimento prévio sobre alumina e água era realizado.

A amperometria de múltiplos pulsos foi a técnica eletroquímica usada na obtenção do voltamograma hidrodinâmico da HQ e de possíveis interferentes (AA e BS). Uma sequência de 12 pulsos de potenciais (de $+0,6$ a 1,7 V, por $70 \mathrm{~ms}$ cada) foi aplicada durante a injeção de soluções padrão de HQ, BS e AA (em triplicata) no sistema BIA. Como o programa usado (GPES versão 4.9 - Metrohm Autolab) permite a aplicação de no máximo 10 pulsos de potencial por experimento, o estudo foi realizado em duas etapas.

A amperometria (acoplada ao sistema BIA) com potencial constante $(+1,00 \mathrm{~V})$ foi usada nas determinações de HQ. As soluções padrão de HQ foram usadas na construção da curva de calibração e preparadas em eletrólito suporte de $\mathrm{H}_{2} \mathrm{SO}_{4} 0,1 \mathrm{~mol} \mathrm{~L}^{-1}$. Amostras de creme dermatológico foram diluídas no mesmo eletrólito antes das injeções. A célula BIA foi preenchida com $150 \mathrm{~mL}$ do mesmo eletrólito antes das injeções. Todas as medidas eletroquímicas foram realizadas à temperatura ambiente, na presença de oxigênio dissolvido.

\section{Análises por cromatografia líquida de alta eficiência (HPLC)}

As medidas em HPLC foram realizadas usando um cromatógrafo da Shimadzu LC-10VP equipado com detector UV/VIS (SPD-10AV), coluna LC (Lychrispher $100 \mathrm{~A}^{\circ} \mathrm{RP} 18-\mathrm{C} 18,250$ x 4,6 mm, $5 \mu \mathrm{m}$ ), forno da coluna (CTO-20A), degaseificador (DGU-20A5), autoamostrador e bomba (LC-10AD-VP). A fase móvel foi composta de acetonitrila e água $(75: 25 \mathrm{v} / \mathrm{v})$ com pH 2,1 (ajustada com ácido fosfórico antes da mistura com a acetonitrila) e a vazão foi de 1,0 $\mathrm{mL} \mathrm{min}^{-1}$. O comprimento de onda do detector UV/VIS foi fixado em $293 \mathrm{~nm}$. As amostras foram simplesmente diluídas na fase móvel antes das injeções.

\section{RESULTADOS E DISCUSSÃO}

Em experimentos preliminares, a oxidação eletroquímica da HQ foi investigada em soluções $\left(0,1 \mathrm{~mol} \mathrm{~L}^{-1}\right.$ cada) de tampão fosfato ( $\mathrm{pH}$ $=7,0)$, tampão acetato $(\mathrm{pH}=4,7)$ e ácido sulfúrico por voltametria cíclica. Estes estudos foram realizados empregando três tipos de materiais como eletrodos de trabalho: BDD, ouro e carbono vítreo (GC). A Figura 1 apresenta o comportamento eletroquímico da HQ e do ácido ascórbico (potencial interferente) com os três eletrodos de trabalho usando a solução de $\mathrm{H}_{2} \mathrm{SO}_{4} 0,1 \mathrm{~mol} \mathrm{~L}^{-1}$ como eletrólito suporte, a qual apresentou melhor desempenho quando comparada com tampão acetato e fosfato, tanto em relação à sensibilidade como seletividade.

Na Figura 1 pode ser observado que a densidade de corrente de oxidação da HQ foi maior quando BDD foi usado como eletrodo de trabalho. O ácido ascórbico (potencial interferente), por sua vez, apresenta pico de oxidação na mesma região de potencial da HQ empregando o eletrodo de GC, enquanto que com os eletrodos de ouro e BDD, o pico de oxidação do ácido ascórbico fica deslocado para potenciais mais positivos, além de apresentar menor corrente de oxidação (comparado com o GC). Nos estudos de estabilidade ou repetibilidade realizados com análises sucessivas de uma solução contendo HQ, tanto por voltametria cíclica como por BIA com detecção amperométrica, o eletrodo de BDD apresentou um desempenho superior aos eletrodos de ouro e GC. Um decréscimo gradativo na corrente de oxidação da HQ foi observado com os eletrodos de ouro e GC, enquanto que com o eletrodo de BDD foi observada uma boa estabilidade em função de sucessivas análises.

O efeito do pré-tratamento ou ativação eletroquímica (catódica ou anódica) sobre o desempenho do eletrodo de BDD também foi estudado. Uma solução contendo $1 \mathrm{mmol} \mathrm{L}^{-1}$ de HQ em meio de 

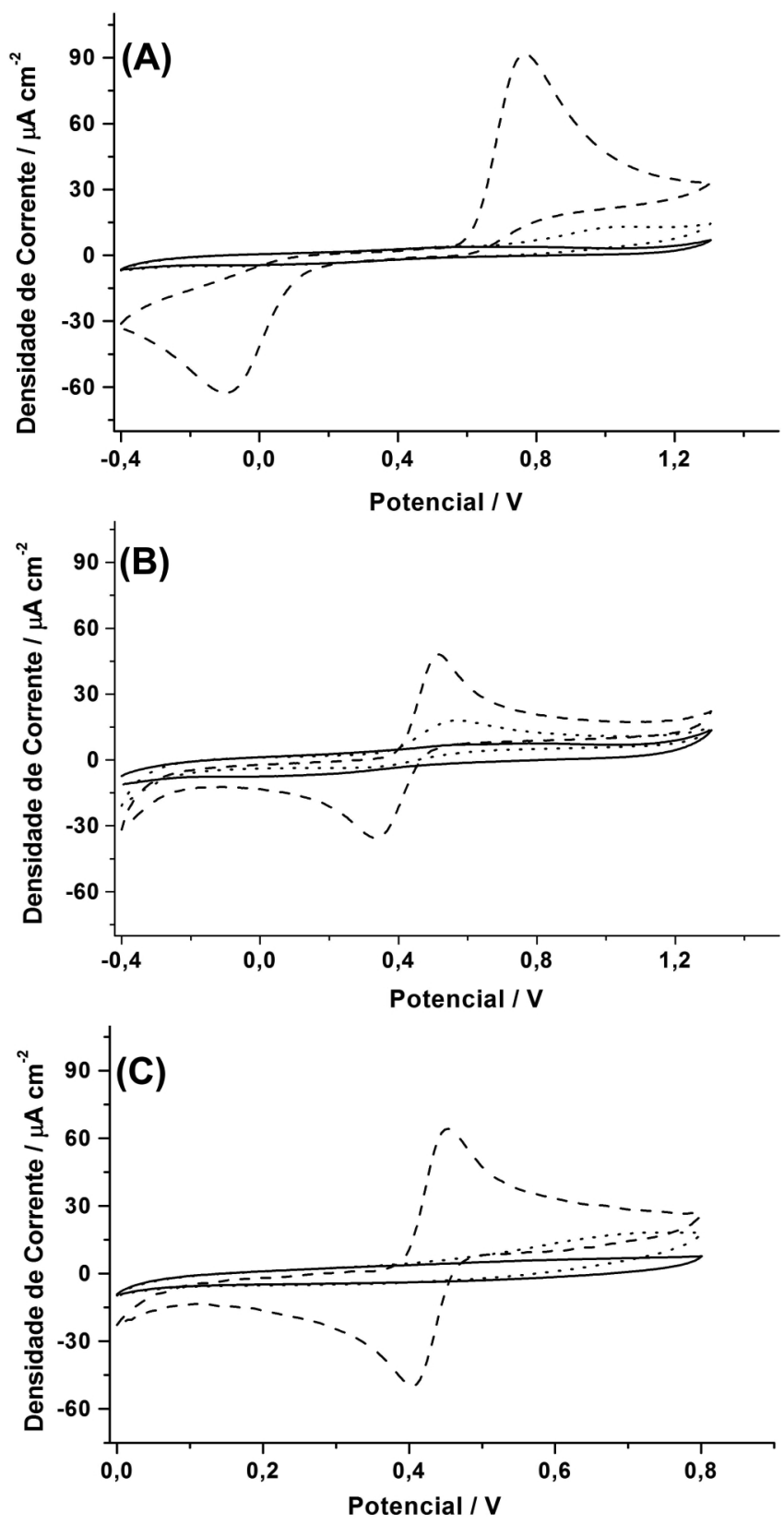

Figura 1. Voltamogramas cíclicos obtidos em meio de $\mathrm{H}_{2} \mathrm{SO}_{4} 0,1 \mathrm{~mol} \mathrm{~L}^{-1} \mathrm{sem}$ (-) e com a adição de 1,0 $\mathrm{mmol} \mathrm{L}^{-1}$ de hidroquinona (----) ou ácido ascórbico (…...). Eletrodos de trabalho: (A) BDD; (B) GC; (C) ouro. Velocidade de varredura de potencial: $50 \mathrm{mV} \mathrm{s}^{-1}$; incremento de potencial: $5 \mathrm{mV}$

$\mathrm{H}_{2} \mathrm{SO}_{4}$,, $1 \mathrm{~mol} \mathrm{~L}^{-1}$ foi analisada após ativação catódica (superfície com terminação de hidrogênio, ou seja, hidrofóbica) e após ativação anódica (superfície com terminação de oxigênio, ou seja, hidrofílica) do eletrodo de BDD. ${ }^{29} \mathrm{O}$ primeiro voltamograma cíclico obtido com o eletrodo de BDD após a ativação anódica gerou uma corrente de oxidação até superior à corrente apresentada na Figura 1A (ativação catódica). No entanto, nos voltamogramas seguintes, uma queda considerável na corrente de oxidação da HQ foi observada (em torno de 50\% no terceiro voltamograma). Provavelmente, o analito (polar) interage mais fortemente com a superfície hidrofílica obtida na ativação anódica, resultando em elevado sinal de corrente no primeiro voltamograma cíclico, mas também promovendo maiores efeitos adsortivos da molécula (HQ) à superfície do eletrodo. Este fenômeno não ocorreu quando a ativação do eletrodo de BDD foi catódica, pois a resposta, apesar de um pouco menor no primeiro voltamograma, se manteve constante após sucessivos voltamogramas cíclicos em meio de solução contendo $1 \mathrm{mmol} \mathrm{L}^{-1}$ de HQ. Em função destes resultados, o eletrodo de BDD com ativação catódica foi adotado como eletrodo de trabalho no restante do estudo.

Como o objetivo deste trabalho era a determinação de HQ empregando BIA com detecção amperométrica (condição não estacionária), também foram realizados estudos para identificar o comportamento eletroquímico da HQ neste sistema. Um voltamograma hidrodinâmico na faixa de potencial que abrange a oxidação eletroquímica da HQ e de possíveis interferentes que podem ser encontrados em formulações farmacêuticas (ácido ascórbico e bissulfito de sódio) foi obtido através da técnica de amperometria de múltiplos pulsos. Uma sequiência de 6 pulsos de potenciais (em duas etapas) de $70 \mathrm{~ms}$ cada $(0,60 ; 0,70 ; 0,80 ; 0,90 ; 1,00 ; 1,10 ; 1,20 ; 1,30 ; 1,40 ; 1,50 ; 1,60$ e 1,70 $\mathrm{V})$ foram aplicados ao eletrodo de BDD posicionado no sistema BIA. A corrente foi monitorada continuamente em cada pulso de potential durante as injeções em triplicata de soluções contendo separadamente $500 \mu \mathrm{mol} \mathrm{L}{ }^{-1}$ de HQ ou $500 \mu \mathrm{mol} \mathrm{L}^{-1}$ de bissulfito de sódio (BS) ou $500 \mu \mathrm{mol} \mathrm{L}{ }^{-1}$ de ácido ascórbico (AA). A Figura 2 apresenta o voltamograma hidrodinâmico resultante, o qual corresponde a um gráfico dos valores médios $(n=3)$ de corrente em função de cada pulso de potencial aplicado.

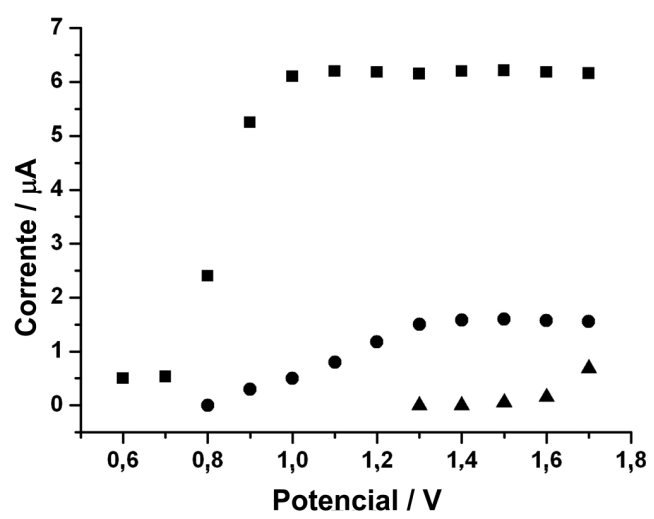

Figura 2. Voltamogramas hidrodinâmicos obtidos com os valores de corrente de pico em função dos pulsos de potenciais aplicados usando BIA com detecção por amperometria de múltiplos pulsos. (ロ) $500 \mu \mathrm{mol} L^{-1}$ de $\mathrm{HQ}$; (•) $500 \mu \mathrm{mol} \mathrm{L} L^{-1}$ de AA; ( $\left.\mathbf{\Delta}\right) 500 \mu \mathrm{mol} \mathrm{L} L^{-1}$ de BS; tempo de aplicação de cada pulso de potencial: $70 \mathrm{~ms}$; eletrólito suporte: $\mathrm{H}_{2} \mathrm{SO}_{4} 0,1 \mathrm{~mol} \mathrm{~L}^{-1}$; velocidade de injeção: $160 \mu \mathrm{L} \mathrm{s}^{-1}$; volume injetado: $100 \mu \mathrm{L}$

Como pode ser observado, a HQ é oxidada a partir de $+0,80 \mathrm{~V}$ e chega a um valor máximo de corrente em $+1,00 \mathrm{~V}$, o que difere um pouco do resultado obtido por voltametria cíclica (sistema estacionário), onde a corrente máxima para oxidação de HQ foi atingida em $+0,8 \mathrm{~V}$. No sistema BIA, um dos interferentes, o AA é oxidado a partir de $+1,00 \mathrm{~V}$ com o valor máximo de corrente em $+1,30 \mathrm{~V}$. O outro possível interferente, o BS, somente é oxidado a partir de $+1,50 \mathrm{~V}$. Essas diferenças de potenciais indicam a viabilidade da determinação de HQ empregando o potencial constante de $+1,00 \mathrm{~V}$ na presença de AA e BS, comumente encontrados em formulações farmacêuticas para impedir a auto-oxidação da HQ. Dessa forma, a aplicação de $+1,00 \mathrm{~V}$ permite a detecção seletiva de HQ numa formulação cosmética contendo AA e/ou BS como antioxidantes. É importante salientar que a determinação seletiva de HQ é possível na presença de ácido ascórbico quando a concentração do mesmo for inferior à de HQ. Quando a concentração de ácido ascórbico for maior do que a da HQ, a determinação seletiva de HQ não será possível com o método proposto.

Os parâmetros do sistema BIA foram otimizados em relação à estabilidade e intensidade de corrente monitorada. Os melhores 
resultados foram obtidos com uma vazão de $160 \mu \mathrm{L} \mathrm{s}^{-1}$ e um volume injetado de $100 \mu \mathrm{L}$ empregando amperometria com potencial constante de 1,00 V. A frequência analítica estimada nestas condições (sem agitação) foi de 70 injeções $\mathrm{h}^{-1}$. Com intuito de aumentar a frequência analítica, estudou-se a possibilidade de incluir na célula BIA um sistema de agitação e, dessa maneira, promover a remoção de forma mais eficiente e rápida da alíquota de amostra ou solução padrão injetada da proximidade da superfície do eletrodo de trabalho. A Figura 3 apresenta o amperograma para injeções de HQ em triplicata no sistema BIA sem e com agitação.



Figura 3. Amperograma obtido para injeções $(n=3)$ de solução contendo $H Q$ $750,0 \mu \mathrm{mol} L^{-1}$ sem e com agitação da solução contida na célula BIA. Potencial

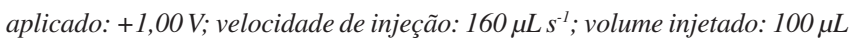

A Figura 3 mostra claramente o aumento de frequência analítica que pode ser alcançado através da inserção do sistema de agitação à célula BIA. Nota-se que os sinais de corrente, na ausência de agitação, requerem um tempo maior para o estabelecimento da linha-base inicial (corrente faradaica igual a zero). Na célula BIA com agitação, verifica-se o rápido restabelecimento da linha-base e, portanto, o número de injeções de soluções padrão ou amostras poderá ser maior em um determinado intervalo de tempo (com agitação), ou seja, a frequência analítica será maior. Com a utilização do sistema de agitação, a frequência analítica foi estimada em 108 injeções $\mathrm{h}^{-1}$. É importante frisar que o sistema de agitação proposto pode ser facilmente implementado fora do ambiente de um laboratório e, portanto, as características portáteis do sistema BIA ficam mantidas. ${ }^{24} \mathrm{~A}$ repetibilidade do sistema é um pouco melhor na ausência de agitação, com o $\mathrm{RSD}=1,1$ e 1,3\% (n=3) no sistema BIA sem e com agitação, respectivamente.

Nas condições otimizadas do sistema BIA (com agitação) foram feitas 20 injeções sucessivas de $30 \mu \mathrm{mol} \mathrm{L}{ }^{-1}$ de HQ (amperograma não mostrado) e verificou-se um desvio padrão relativo (RSD) de $0,45 \%$ $(n=20)$. Valores tão baixos de RSD foram obtidos em trabalhos anteriores empregando BIA, devido à elevada precisão da injeção obtida devido ao uso da pipeta eletrônica. ${ }^{23-25}$ Posteriormente, a faixa linear do método amperométrico foi avaliada através de injeções de soluções padrão contendo concentrações crescentes de HQ. A Figura 4 apresenta o amperograma obtido neste estudo e a respectiva curva de calibração.

Nota-se que o método amperométrico proposto apresenta uma ampla faixa linear de resposta (10 a $2000 \mu \mathrm{mol} \mathrm{L} \mathrm{L}^{-1}$ de HQ), com a seguinte equação de reta: $\mathrm{i}(\mu \mathrm{A})=-0,670+0,123 \mathrm{C}\left(\mu \mathrm{mol} \mathrm{L} \mathrm{L}^{-1}\right)$ e coeficiente de correlação igual a 0,9999. Os limites de detecção e quantificação foram estimados em $0,016 \mathrm{e} 0,055 \mu \mathrm{mol} \mathrm{L}^{-1}$, respectivamente. A ampla faixa linear do método amperométrico e a elevada sensibilidade permitem que amostras com variadas concentrações de HQ possam
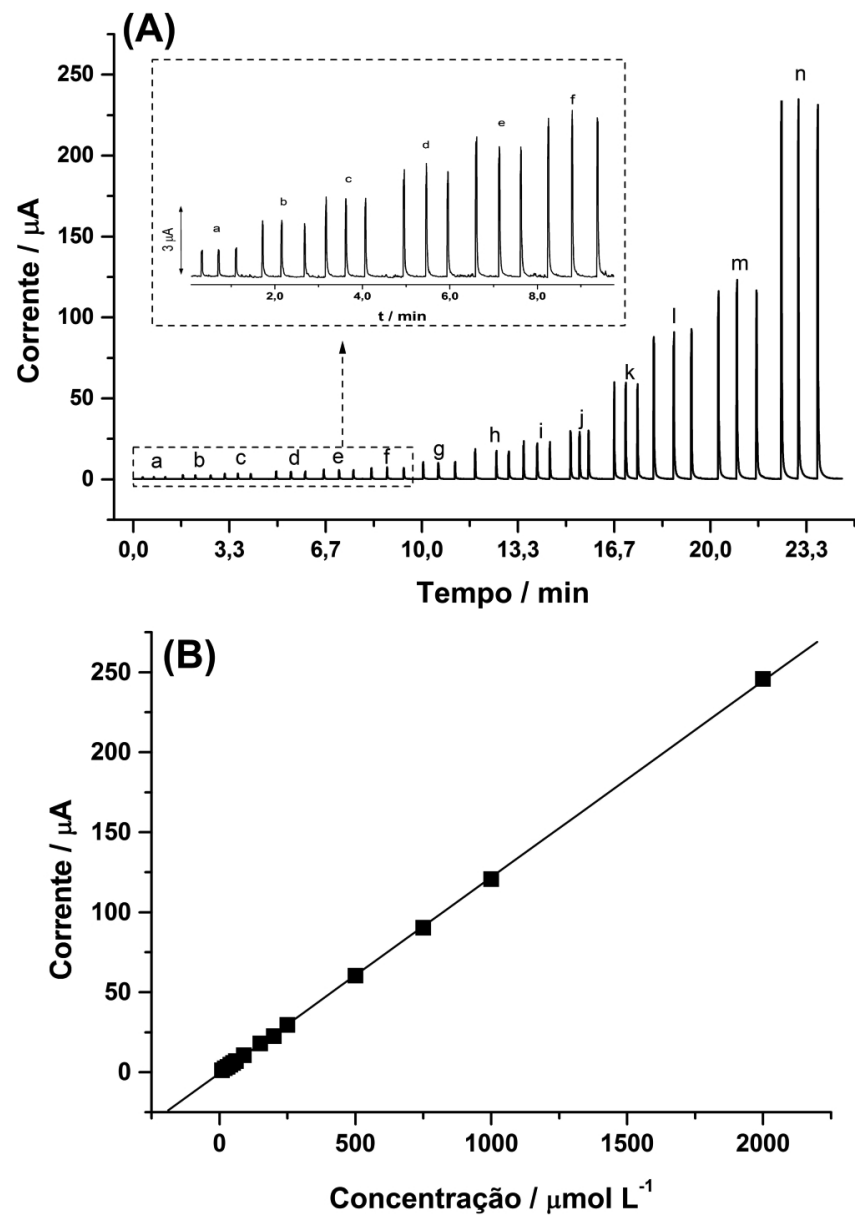

Figura 4. (A) Amperograma obtido a partir de injeções de soluções contendo concentrações crescentes de $H Q\left(\mu m o l L^{-1}\right)$ usando pipeta eletrônica: $a=$ $10 ; b=20 ; c=30 ; d=40 ; e=50 ; f=60 ; g=90 ; h=150 ; i=200 ; j=$ 250; $k=500 ; l=750 ; m=1000 ; n=2000$. Potencial aplicado: $+1,00 \mathrm{~V}$; velocidade de injeção: $160 \mu \mathrm{L} \mathrm{s} \mathrm{s}^{-1}$; volume injetado: $100 \mu \mathrm{L}$. (B) Respectiva curva de calibração $(R=0,999)$

ser analisadas sem prévias diluições ou pré-concentrações.

A Figura 5 apresenta um estudo sobre a eficiência de lavagem e efeito de memória na superfície do eletrodo de trabalho no interior da célula BIA, frente a injeções sucessivas de soluções contendo HQ em concentrações discrepantes em uma ordem de grandeza (50 e $500 \mu \mathrm{mol} \mathrm{L}-1)$.

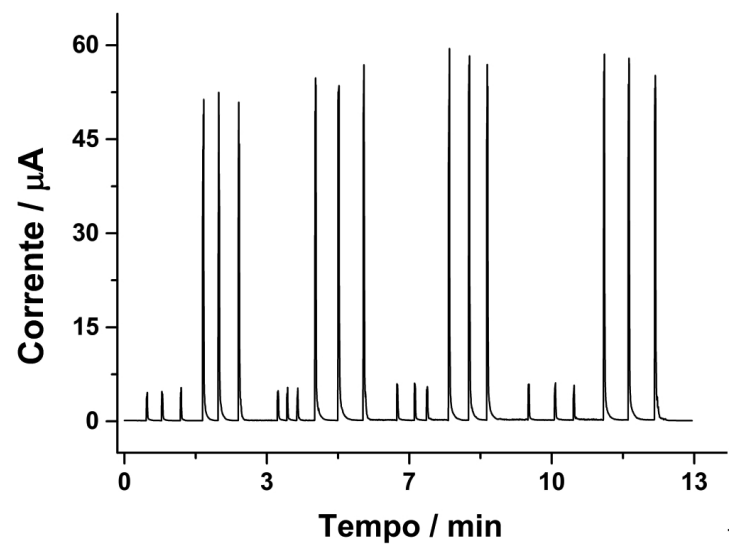

Figura 5. Amperograma obtido para injeções $(n=3)$ intercaladas de solução contendo HQ 50,0 e 500,0 $\mu \mathrm{mol} L^{-1}(n=12)$. Potencial aplicado: $+1,00 \mathrm{~V}$;

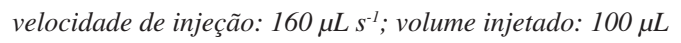


No amperograma da Figura 5 nota-se que as correntes de oxidação referentes a $50 \mu \mathrm{mol} \mathrm{L}^{-1}$ de HQ possuem valores de corrente bastante próximos $(\mathrm{RSD}=2,3 \% ; \mathrm{n}=12)$, assim como as correntes referentes à solução contendo $500 \mu \mathrm{mol} \mathrm{L}{ }^{-1}$ de HQ $(\mathrm{RSD}=1,9 \%$; $\mathrm{n}=12)$, apesar das injeções alternadas de soluções com concentração de HQ tão discrepantes. Portanto, verifica-se que o eletrodo de BDD acoplado ao sistema BIA com agitação não apresenta efeito de memória.

$\mathrm{O}$ método proposto para determinação de HQ foi empregado na análise de duas amostras de formulações cosméticas industrializadas, sendo que estas foram dopadas com BS e AA em nível de concentração semelhante ao encontrado em formulações manipuladas contendo tais antioxidantes (100:3:10 m/m para HQ, AA e BS, respectivamente). Com intuito de comparar os resultados obtidos com o método proposto, as amostras também foram analisadas por HPLC. Os resultados são apresentados na Tabela 1.

Tabela 1. Comparação dos resultados obtidos pelo método amperométrico com os obtidos por HPLC

\begin{tabular}{ccc}
\hline Amostra & $\begin{array}{c}\text { BIA } \pm \text { SD } \\
(\mathrm{mg} \mathrm{HQ} / \mathrm{g})\end{array}$ & $\begin{array}{c}\mathrm{HPLC} \pm \mathrm{SD} \\
(\mathrm{mg} \mathrm{HQ} / \mathrm{g})\end{array}$ \\
\hline A1 & $29 \pm 1$ & $30 \pm 1$ \\
$\mathrm{~A} 2$ & $30 \pm 1$ & $31 \pm 1$ \\
\hline
\end{tabular}

O método proposto apresentou resultados similares aos obtidos por HPLC. Considerando o teste $t$, os resultados médios $(n=3)$ de ambos os métodos não apresentaram diferença significativa em um nível de confiança de $95 \%$, sendo que o $t_{\text {calculado }}$ foi inferior ao $t_{\text {tabelado }}$ $(2,78)$. A similaridade dos resultados obtidos entre o método proposto e HPLC demonstra que os demais componentes da matriz, assim como AA e BS (adicionados à amostra), não são considerados interferentes na determinação de HQ com o método proposto. Adicionalmente, também foram realizados estudos de adição e recuperação. Três diferentes níveis de concentração de HQ foram adicionados às amostras analisadas (após adequada diluição) e em todos os casos, os valores de recuperação observados se situaram na faixa entre 90 e $96 \%$.

Os resultados apresentados até aqui demonstram que o método proposto é simples, rápido e preciso na determinação de HQ. No entanto, a necessidade do uso de uma pipeta eletrônica no procedimento de injeção do sistema BIA, além de aumentar o custo da análise, pode limitar seu uso no dia a dia, pois pipetas eletrônicas não são normalmente encontradas em laboratórios comuns de controle de qualidade. Em função disto, para aumentar a probabilidade de uso e diminuir ainda mais o custo do método proposto, estamos incluindo neste trabalho uma opção ao uso de pipeta eletrônica no procedimento de injeção do sistema BIA. Na Figura 6 é apresentado um amperograma e respectiva curva de calibração obtidos com a injeção de soluções contendo HQ usando uma seringa descartável em substituição à pipeta eletrônica usada anteriormente (Figura 3). É importante salientar que a seringa é descartável para seu uso original (aplicação de insulina), mas pode ser usada inúmeras vezes para o fim aqui proposto.

Como pode ser observado, foi obtido um resultado similar ao apresentado na Figura 3 (uso de pipeta eletrônica). O coeficiente de correlação foi calculado em 0,999 . Nestas condições, também foi efetuado um estudo de repetibilidade com a obtenção de um RSD = $4,9 \%(n=10)$, o qual é levemente superior ao $\operatorname{RSD}(\sim 2,0 \%)$ calculado quando a pipeta eletrônica foi usada no procedimento de injeção. É importante salientar que os resultados apresentados empregando a seringa descartável no procedimento de injeção foram obtidos por um analista com certa experiência com sistemas BIA, o que pode ser considerado uma desvantagem, pois a precisão na injeção independe do operador se a pipeta eletrônica é empregada. O uso da seringa
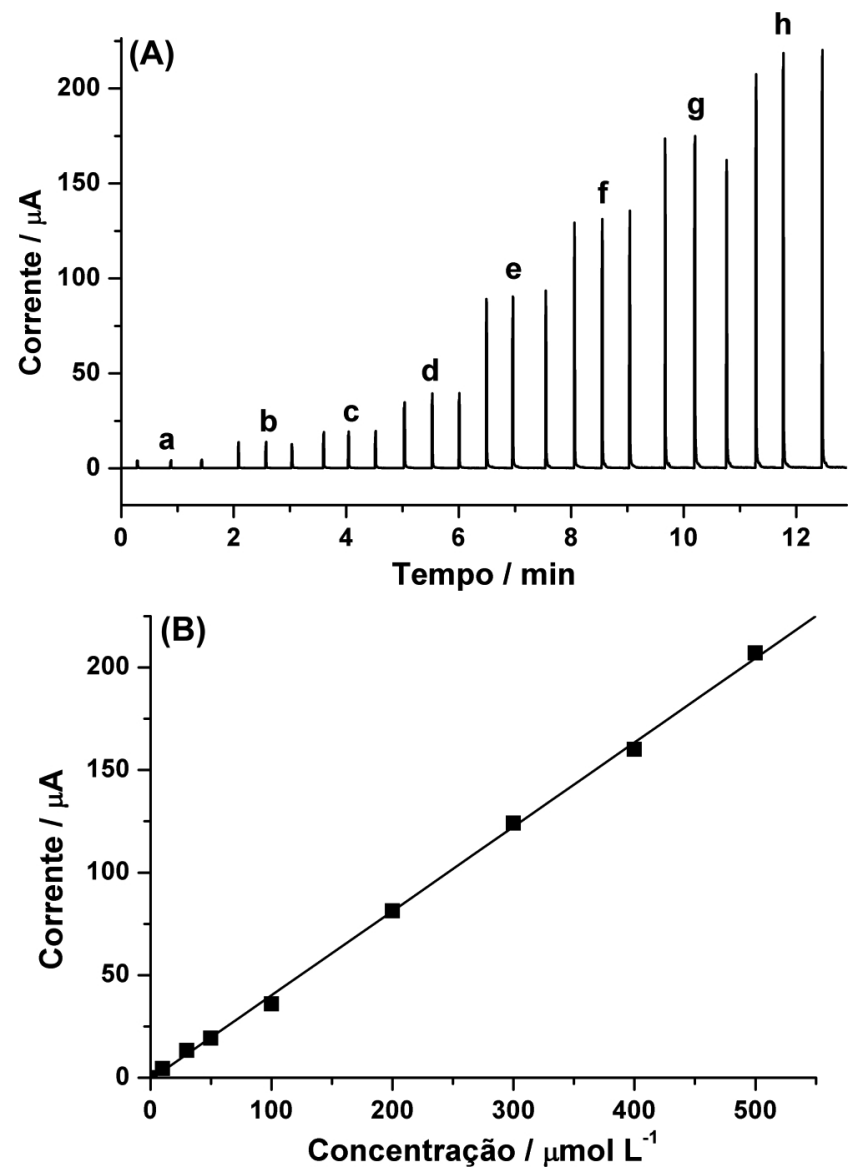

Figura 6. (A) Amperograma obtido a partir de injeções de soluções contendo concentrações crescentes de $H Q\left(\mu \mathrm{mol} L^{-1}\right)$ usando seringa descartável: $a=$ $10 ; b=30 ; c=50 ; d=100 ; e=200 ; f=300 ; g=400 ; h=500$. Potencial aplicado: $+1,00 \mathrm{~V}$; volume injetado: $150 \mu \mathrm{L}$. (B) Respectiva curva de calibração $(R=0,999)$

descartável diminui consideravelmente o custo do método, mas a precisão passa a ser dependente da eficiência do operador no procedimento de injeção. Uma alternativa para contornar este problema seria a inclusão de um padrão interno no sistema de análise, conforme sugerido anteriormente. ${ }^{31,32}$ No entanto, no sistema proposto isto não é possível, pois o produto de oxidação da HQ é detectado em potenciais mais negativos onde o padrão interno poderia ou deveria ser constantemente monitorado (amperometria de múltiplos pulsos).

Na Tabela 2 é apresentada uma comparação entre as características analíticas do método proposto para determinação de hidroquinona com outros métodos que empregam detecção eletroquímica na literatura. ${ }^{33-40}$ Verifica-se que o método proposto apresentou a mais ampla faixa de trabalho e o menor limite detecção em comparação aos demais métodos eletroanalíticos, além da elevada frequência analítica típica dos métodos em fluxo, sobretudo empregando a técnica BIA.

\section{CONCLUSÕES}

Os resultados apresentados demonstram que o método proposto que emprega sistema BIA acoplado à detecção amperométrica usando eletrodo de BDD possui grande potencial para ser empregado na determinação de HQ em formulações cosméticas. O método proposto pode ser aplicado à determinação seletiva de $\mathrm{HQ}$ na presença de BS e AA (antioxidantes comumente encontrados em tais formulações), sendo que apresenta ampla faixa linear de trabalho e elevada sensibilidade. A associação do sistema BIA com a detecção amperométrica (sob 
Tabela 2. Comparação entre as características analíticas do método proposto para determinação de HQ com trabalhos da literatura empregando deteç̧ão eletroquímica

\begin{tabular}{|c|c|c|c|c|c|}
\hline Eletrodo de trabalho & Técnica & $\begin{array}{c}\text { Faixa linear } \\
\mathrm{mol} \mathrm{L}^{-1}\end{array}$ & $\begin{array}{l}\mathrm{LD} \\
\mathrm{mol} \mathrm{L}^{-1}\end{array}$ & $\begin{array}{c}\text { FA } \\
\text { análises } \mathrm{h}^{-1}\end{array}$ & Ref. \\
\hline Biossensor & SWV & 0,25 a $5,5 \times 10^{-3}$ & $2 \times 10^{-6}$ & NI & 34 \\
\hline Biossensor & AMP & 0,075 a $1,6 \times 10^{-3}$ & $8,1 \times 10^{-6}$ & NI & 35 \\
\hline BDD & DPV & 0,05 a $7,0 \times 10^{-3}$ & $1,7 \times 10^{-6}$ & NI & 36 \\
\hline Biossensor & $\mathrm{CV}$ & 0,06 a $8,9 \times 10^{-3}$ & $8,3 \times 10^{-6}$ & NI & 37 \\
\hline Biossensor & DPV & 0,25 a $2,4 \times 10^{-3}$ & $2,5 \times 10^{-5}$ & NI & 38 \\
\hline Biossensor & LSV & 0,25 a $4,0 \times 10^{-3}$ & $3,8 \times 10^{-5}$ & NI & 39 \\
\hline $\mathrm{CE}$ & DPV & 0,001 a $0,1 \times 10^{-3}$ & $0,9 \times 10^{-6}$ & NI & 40 \\
\hline BDD & AMP & 0,01 a $2,0 \times 10^{-3}$ & $0,02 \times 10^{-6}$ & 108 & Este trabalho \\
\hline
\end{tabular}

CE: eletrodo de compósito; SWV: voltametria de onda quadrada; AMP: amperometria; DPV: voltametria de pulso diferencial; CV: voltametria cíclica; LSV: voltametria de varredura linear; LD: limite de detecção; FA: frequência analítica; NI: não informado; Ref.: referência.

agitação mecânica) faz com que o método analítico seja rápido ( 108 injeções $\left.\mathrm{h}^{-1}\right)$, de fácil execução e com reduzido consumo de reagentes e amostras por análise. Adicionalmente, o sistema proposto pode ser facilmente empregado em determinações em campo, pois o sistema BIA proposto, assim como alguns micropotenciostatos disponíveis comercialmente têm características portáteis.

\section{AGRADECIMENTOS}

Ao CNPq (476269/2010-5, 478081/2010-3 e 305227/2010-6), FAPEMIG (APQ-01430-11 e 01856-10) e CAPES pelo suporte financeiro.

\section{REFERÊNCIAS}

1. Tagliari, M. P.; Stulzer, H. K.; Kelmann, R. G.; Kuminek, G.; Silva, M. A. S.; Cosmet. Toilet 2008, 20, 50.

2. Nathaly, C. G.; Sandra, S.; Noemi, N.; Quim. Nova 2011, 34, 635.

3. Pistonesi, M. F.; Di Nezio, M. S.; Centurion, M. E.; Lista, A. G.; Fragroso, W. D.; Pontes, M. J. C.; Araujo, M. C. U.; Fernandes Band, B. S.; Talanta 2010, 83, 320.

4. Firth, J.; Rix, I.; Analyst 1986, 111, 129.

5. Sharma, O. P.; Bhat, T. K.; Singh, B.; J. Chromatogr. 1998, 822, 167.

6. Sakodinskaya, I. K.; Desiderio, C.; Nardi, A.; Fanali,, S.; J. Chromatogr. 1992, 596, 95

7. Zhimin, L.; Zhenling, W.; Yanyan, C.; Sens. Actuators, B 2011, 157, 540.

8. Saleh, A. A. J.; Mahbubur, M. R.; Guang-Ri, X.; Electrochim. Acta 2011, 56,5266

9. Zhaohui, H.; Yanli, Z.; Qin, L.; Microchim. Acta 2011, 173, 119.

10. Dong-Ming, Z.; Xiu-Hua, Z.; Li-Jun, F.; Colloids Surf., B 2009, 74, 317.

11. Ping, Y.; Qiyong, Z.; Yonghong, C.; J. Appl. Polym. Sci. 2009, 113, 2881.

12. Bo, K.; Tanji, Y.; Xiaoying, L.; Anal. Lett. 2007, 40, 2141.

13. Liang, W.; Pengfei, H.; Junyue, B.; Int. J. Electrochem. Sci. 2007, 2, 123.

14. Qi, H. L.; Zhang, C. X.; Electroanalysis 2005, 17, 832.

15. Nasr, B.; Abdellatif, G.; Canizares, P.; Environ. Sci. Technol. 2005, 39, 7234.

16. Sopchak, D.; Miller, B.; Avyigal, Y.; J. Electroanal. Chem. 2002, 538, 39.

17. Ramesham, R.; Rose, M. F.; J. Mater. Sci. Lett. 1997, 16, 799.

18. Salazar-Banda, G. R.; Andrade, L. S.; Nascente, P. A. P.; Pizani, P. S.; Rocha-Filho, R. C.; Avaca, L. A.; Electrochim. Acta 2006, 51, 4612.
19. de Barros, R. D. M.; Ribeiro, M. C.; An-Sumodjo, P. T.; Juliao, M. S. D.; Serrano, S. H. P.; Ferreira, N. G.; Quim. Nova 2005, 28, 325.

20. Peckova, K.; Musilova, J.; Barek, J.; Crit. Rev. Anal. Chem. 2009, 39, 148.

21. Quintino, M. S. M.; Angnes, L.; Electroanalysis 2004, 16, 513.

22. Pereira, P. F.; Marra, M. C.; Munoz, R. A. A.; Richter, E. M.; Talanta 2012, 90, 99.

23. Silva, R. A. B.; Gimenes, D. T.; Tormin, T. F.; Munoz, R. A. A.; Richter, E. M.; Anal. Methods 2011, 3, 2804.

24. Silva R. A. B.; Montes, R. H. O.; Richter, E. M.; Munoz, R. A. A.; Food Chem. 2012, 133, 200.

25. Tormin, T. F.; Gimenes, D. T.; Richter, E. M.; Munoz, R. A. A.; Talanta 2011, 85, 1274 .

26. Pedrotti, J. J.; Angnes, L.; Gutz, I. G. R.; Electroanalysis 1996, 8, 673.

27. Terashima, C.; Rao, T. N.; Sarada, B. V.; Kubota, Y.; Fujishima, A.; Anal. Chem. 2003, 75, 1564.

28. Gimenes, D. T.; De Freitas, J. M.; Munoz, R. A. A.; Richter, E. M.; Electroanalysis 2011, 23, 2521.

29. Suffredini, H. B.; Pedrosa, V. A.; Codognoto, L.; Machado, S. A. S.; Rocha-Filho, R. C.; Avaca, L. A.; Electrochim. Acta 2004, 49, 4021.

30. Miranda, J. A. T.; Cunha, R. R.; Gimenes, D. T.; Munoz, R. A. A.; Richter, E. M.; Quim. Nova 2012, 35, 1459.

31. Gimenes, D. T.; Pereira, P. F.; da Silva, R. A. B.; Cunha, R. R.; Munoz, R. A. A.; Richter, E. M.; Electroanalysis 2012, 24, 1805.

32. Gimenes, D. T.; Santos, W. T. P.; Munoz, R. A. A.; Richter, E. M.; Electrochem. Commun. 2010, 12, 216.

33. García, P. L.; Santoro, M. I. R.; Kedor-Hackmann, E. R. M.; Singh, A. K.; J. Pharm. Biomed. Anal. 2005, 40, 490.

34. de Oliveira, I. R. W. Z.; Vieira, I. C.; Enzyme Microb. Technol. 2005, 38, 449.

35. Vieira, I. C.; Fatibello-Filho, O.; Talanta 2000, 52, 681.

36. Zhao, G. H.; Tang, Y. T.; Liu, M. C.; Lei, Y. Z.; Xiao, X. E.; Chin. J. Chem. 2007, 25, 1445.

37. Vieira, I. C.; Fatibello-Filho, O.; Angnes, L.; Anal. Chim. Acta 1999, 398, 145.

38. De Oliveira, I. R. W. Z.; Vieira, I. C.; Lupetti, K. O.; Fatibello-Filho, O.; de Favere, V. T.; Laranjeira, M. C. M.; Anal. Lett. 2004, 37, 3111.

39. Lupetti, K. O.; Zanotto-Neto, G.; Fatibello-Filho, O.; J. Braz. Chem. Soc. 2006, 17, 1329.

40. Mendes, R. K.; Cervini, P.; Cavalheiro, E. T. G.; Talanta 2005, 68, 708. 